\title{
Optimal Interpolation of Translation Operator in Multilevel Fast Multipole Algorithm
}

\author{
Özgür Ergül, Student Member, IEEE, and Levent Gürel, Senior Member, IEEE
}

\begin{abstract}
Lagrange interpolation of the translation operator in the three-dimensional multilevel fast multipole algorithm (MLFMA) is revisited. Parameters of the interpolation, namely, the number of interpolation points and the oversampling factor, are optimized for controllable error. Via optimization, it becomes possible to obtain the desired level of accuracy with the minimum processing time.
\end{abstract}

Index Terms-Lagrange interpolation, multilevel fast multipole algorithm, translation operator.

\section{INTRODUCTION}

$\mathbf{T}$ HE multilevel fast multipole algorithm (MLFMA) [1], [2] requires translations to convert the radiated fields of the basis clusters into incoming waves for the testing clusters. In a matrix-vector multiplication, translations are performed between the clusters that are at the same level but far from each other. Through the factorization of the Green's function, translation operators are independent from the radiation and receiving patterns of the basis and testing clusters, respectively [3]. To be employed repeatedly, these operators are calculated and stored in the memory before the iterations.

Since direct calculation of the translation operators requires $O\left(N^{3 / 2}\right)$ operations, where $N$ is the number of unknowns, processing time for their setup increases rapidly and becomes substantial as problem size grows. As a remedy, a two-step computation is suggested based on the interpolation of the translation operator [4]: First, the translation operator is expressed as a band-limited function of a variable $\varphi$ and it is sampled at $O(N)$ points with respect to this variable. Second, the operator is evaluated at the required points by interpolation from the previous samples. With an efficient interpolation algorithm, processing time for the calculation of the translation operators is reduced to $O(N)$.

In [4], Lagrange interpolation was proposed to efficiently fill the translation matrices for large problems. However, the parameters of the interpolation, namely, the number of interpolation points and the oversampling factor, were fixed. With the parameters fixed, the interpolation error is not controllable and

Manuscript received May 30, 2006; revised August 17, 2006. This work was supported by the Scientific and Technical Research Council of Turkey (TUBITAK) under Research Grant 105E172, by the Turkish Academy of Sciences in the framework of the Young Scientist Award Program (LG/TUBA-GEBIP/2002-1-12), and by contracts from ASELSAN and SSM.

The authors are with the Department of Electrical and Electronics Engineering, Bilkent University, TR-06800, Bilkent, Ankara, Turkey (e-mail: ergul@ ee.bilkent.edu.tr; lgurel@bilkent.edu.tr).

Digital Object Identifier 10.1109/TAP.2006.886562 the processing time is not minimized. In this paper, we revisit the Lagrange interpolation of the translation operators and optimize the parameters of the interpolation to obtain the desired level of accuracy with minimum processing time. The optimal parameters are compared to the fixed parameters to demonstrate the improvement obtained with the optimization.

\section{LAGRANGE INTERPOLATION OF THE TRANSLATION OPERATORS}

A three-dimensional (3-D) translation operator between a pair of basis and testing clusters at the same level can be written as

$$
T(k, \hat{k}, \boldsymbol{D})=\frac{i k}{4 \pi} \sum_{l=0}^{L}(i)^{l}(2 l+1) h_{l}^{(1)}(k D) P_{l}(\hat{\boldsymbol{k}} \cdot \hat{\boldsymbol{D}})
$$

where $h_{l}^{(1)}$ is the spherical Hankel function of the first kind, $P_{l}$ is the Legendre polynomial, $k$ is the wavenumber, and $\hat{k}$ is a unit vector representing the angular directions. The centers of the basis and testing clusters are separated by the vector $\boldsymbol{D}$, where

$$
\boldsymbol{D}=\hat{\boldsymbol{D}} D \text {. }
$$

The summation in (1) is truncated at $L$, where $L$ is the number of multipoles required to accurately represent the spectral contents of both the translation operator and the related radiation and receiving patterns. Considering cubic clusters with edges $a$ and using the excess bandwidth formula [5] for the worst case scenario [6]

$$
L \approx 1.73 k a+2.16\left(d_{0}\right)^{2 / 3}(k a)^{1 / 3}
$$

where $d_{0}$ is the desired number of digits of accuracy.

In Fig. 1(a), the truncation number $L$ is plotted with respect to $d_{0}$ and for different values of the cluster size $a$ increasing by a factor of two from $0.25 \lambda$ to $64 \lambda$, where $\lambda$ is the wavelength. For any problem, $0.25 \lambda$ corresponds to the size of the clusters at the lowest level of the multilevel tree structure. On the other hand, the size of the largest clusters depends on the size of the problem. Fig. 1(a) demonstrates that $L$ grows rapidly as the cluster size increases. For a fixed $a$, however, $L$ increases gradually with respect to $d_{0}$ and the variation is small for large $a$.

Processing time required to calculate the translation operator in (1) is measured on a 1.8-GHz 64-bit Opteron-244 processor. In Fig. 1(b), the processing time is plotted with respect to the same parameters as in Fig. 1(a). The values are given for a single interaction between a pair of basis and testing clusters while a typical problem requires the calculation of numerous cluster-cluster interactions. Since $L=O(k a)$, the processing 


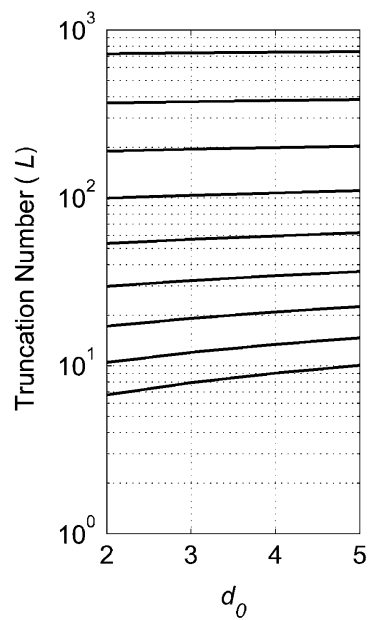

(a)

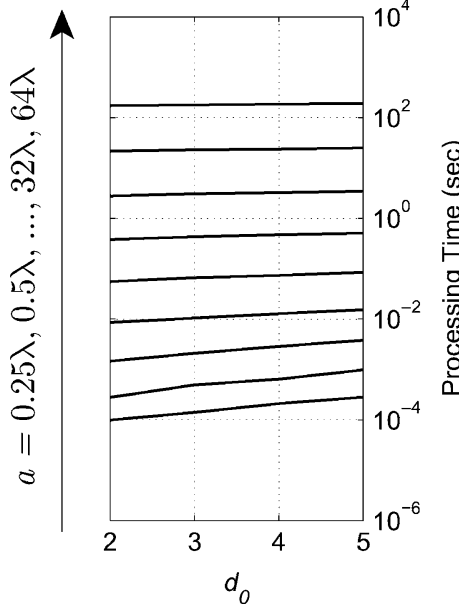

(b)

Fig. 1. (a) Truncation number as a function of $d_{0}$ and the cluster size $a$. (b) Processing time to compute the translation function for a single cluster-cluster interaction. In both figures, there are nine curves for different values of the cluster size increasing by a factor of two from $0.25 \lambda$ to $64 \lambda$. The lowest and highest curves correspond to $0.25 \lambda$ and $64 \lambda$, respectively.

time to evaluate (1) for a fixed $\hat{\boldsymbol{k}}$ is $O(k a)$. In addition, the number of angular directions $\hat{\boldsymbol{k}}$ is $O\left(L^{2}\right)$ and the processing time to evaluate (1) becomes $O\left(k^{3} a^{3}\right)$ for a cluster-cluster interaction. For low levels of MLFMA, $O\left(k^{3} a^{3}\right)=O(1)$, which is acceptable although the number of clusters in these levels is $O(N)$. However, for the largest clusters of a problem, $O(k a)=$ $O\left(N^{1 / 2}\right)$ and $O\left(k^{3} a^{3}\right)=O\left(N^{3 / 2}\right)$. Therefore, as $N$ becomes large, the processing time required to calculate the translation operators for a problem is dominated by the evaluations for the high-level clusters, although the number of these clusters is $O(1)$. In addition, the setup time for the translation matrix becomes dominant compared to the time required for other parts of MLFMA, even the matrix-vector multiplications that can be performed in $O(N \log N)$ time.

Defining the variable $\varphi=\cos ^{-1}(\hat{\boldsymbol{k}} \cdot \hat{\boldsymbol{D}})$, the translation operator can be expressed as a band-limited function of $\varphi[4]$ as

$$
T(k, D, \varphi)=\frac{i k}{4 \pi} \sum_{l=0}^{L}(i)^{l}(2 l+1) h_{l}^{(1)}(k D) P_{l}(\cos \varphi) .
$$

Choosing an oversampling factor $s$ and sampling the operator along $\varphi$ from 0 to $2 \pi$ at $\lfloor s L\rfloor=O(N)$ equally spaced points $(\lfloor\cdot\rfloor$ represents the floor operation), i.e., at $\varphi_{i}=2 \pi(i-1) /(\lfloor s L\rfloor-1)$ and $i=1, \ldots,\lfloor s L\rfloor$, the translation operator can be obtained by Lagrange interpolation at any point as

$$
\tilde{T}(k, D, \varphi)=\sum_{i=f+1-p}^{f+p} T\left(k, D, \varphi_{i}\right) w_{i}(\varphi)
$$

where $\tilde{T}$ represents the translation function perturbed by the interpolation error

and

$$
f=\left\lfloor\frac{\varphi(\lfloor s L\rfloor-1)}{2 \pi}+1\right\rfloor
$$

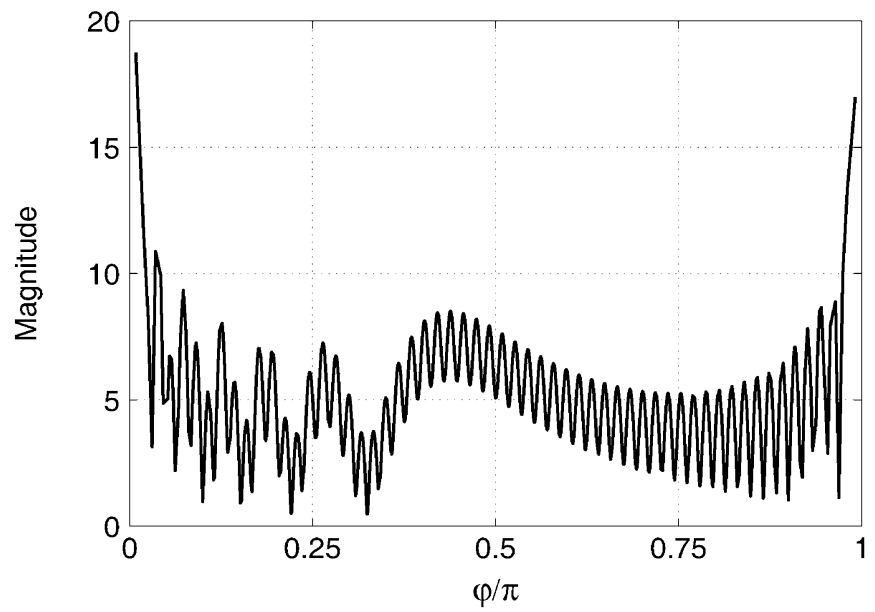

(a)

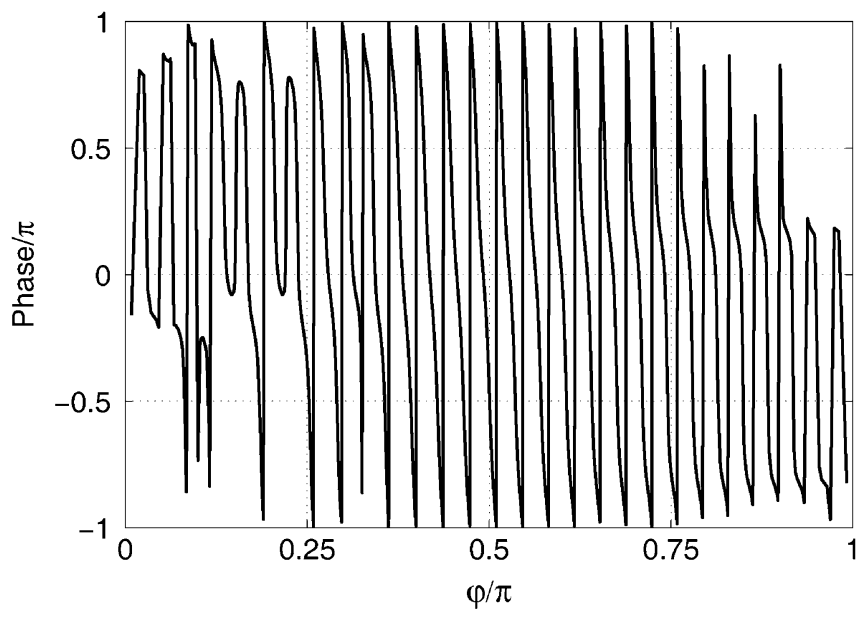

(b)

Fig. 2. (a) Magnitude and (b) phase of the translation function with respect to $\varphi$ for the case of $a=4 \lambda, d_{0}=3$, and $\boldsymbol{D}=\hat{\boldsymbol{x}} 2 a$.

$$
w_{i}(\varphi)=\prod_{\substack{j=f+1-p \\ j \neq i}}^{f+p} \frac{\varphi-\varphi_{j}}{\varphi_{i}-\varphi_{j}} .
$$

In (5) and (7), $p$ is the number of interpolation points employed at each side of the target location $\varphi$.

\section{OPTIMAL INTERPOLATION}

Fig. 2(a) and (b) depicts the magnitude and phase of the translation operator, respectively, for two clusters separated by $\boldsymbol{D}=$ $\hat{x} 2 a$, where $a=4 \lambda$. The number of accurate digits $d_{0}$ is 3 and $L=57$. We perform the direct calculation of the translation operator, where the function is evaluated at the required points by using (4). In the $\phi$ direction, there are $2(L+1)=116$ samples that are equally spaced from 0 to $2 \pi$. In the $\theta$ direction, there are $(L+1)=58$ samples (zeros of the Legendre polynomial) and they are not equally spaced. Then, there are a total of $2(L+1)^{2}=6728$ distinct $\hat{\boldsymbol{k}}$ directions to evaluate the translation operator. It should be noted that the transform from (1) to (4) not only depends on $L$, but also on the relative positions of the clusters, i.e., it also depends on $\boldsymbol{D}$. 


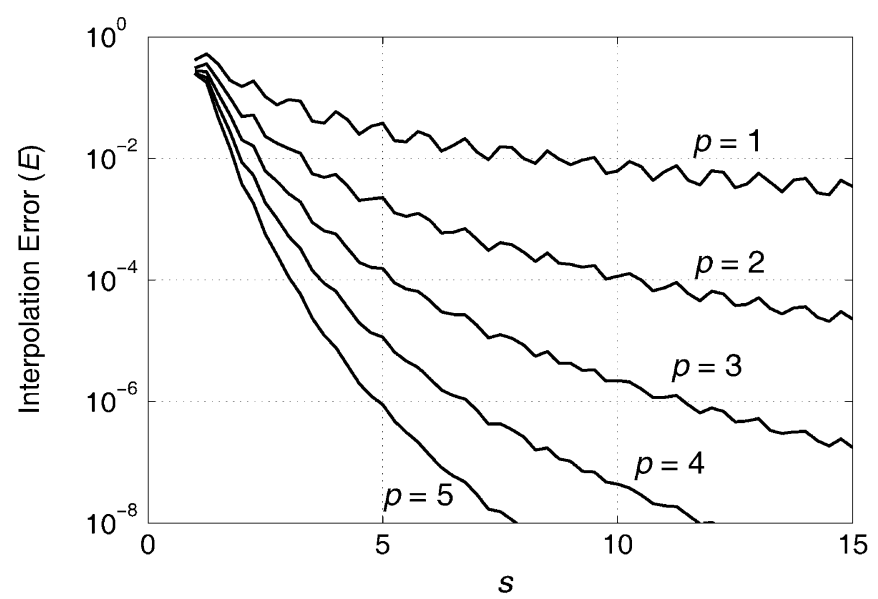

(a)

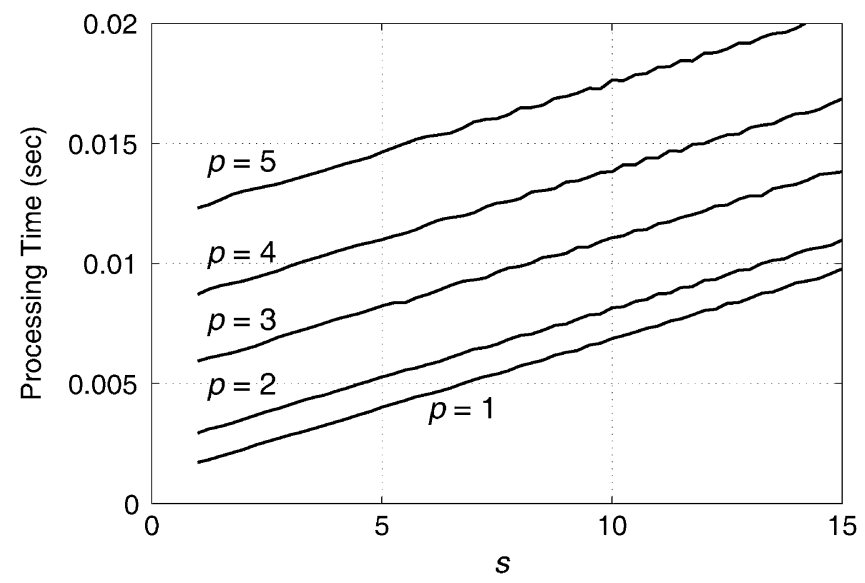

(b)

Fig. 3. (a) Interpolation error and (b) processing time with respect to interpolation parameters $p$ and $s$ for the translation function in Fig. 2.

Before the translation matrix is filled via Lagrange interpolation, the parameters $s$ and $p$ must be determined. For fixed values of $d_{0}$ and $a$, we perform a scan over the $s$ and $p$ parameters to find their optimal values. Fig. 3(a) demonstrates the interpolation error with respect to $s$ and $p$ for the case in Fig. 2. The interpolation error is defined as

$$
E=\max \left\{E_{n}\right\}, \quad E_{n}=\frac{\left|\tilde{T}\left(\varphi_{n}\right)-T\left(\varphi_{n}\right)\right|}{\max _{\varphi}\{|T(\varphi)|\}}
$$

where $n=1, \ldots, 2(L+1)^{2}$ and $\varphi_{n}$ represents the sampling points. The interpolation error decreases when $p$ or $s$ is increased. In this case $d_{0}=3$, which means that MLFMA computes the interactions with three digits of accuracy. Thus, $(p, s)$ pairs leading to larger than $10^{-3}$ error are not allowable. In other words, the error introduced by the interpolation of the translation operator should be adjusted according to the desired level of accuracy.

This strategy yields a set of $(p, s)$ pairs satisfying the error criterion. Optimization is completed by choosing the $(p, s)$ pair with the minimum processing time. As shown in Fig. 3(b), processing time (measured on a $1.8-\mathrm{GHz} 64$-bit Opteron-244 processor) to evaluate the translation operator increases as $p$ or $s$ is
TABLE I

Speedup Obtained By Using the Optimal $(p, s)$ PAIR For $a \geq 4 \lambda$

\begin{tabular}{|c|c|ccccc|}
\hline$d_{0}$ & $(p, s)$ & $a=4 \lambda$ & $a=8 \lambda$ & $a=16 \lambda$ & $a=32 \lambda$ & $a=64 \lambda$ \\
\hline \hline 2 & $(2,3.5)$ & 14.0 & 27.5 & 54.3 & 108.3 & 216.0 \\
\hline 3 & $(2,6.5)$ & 10.8 & 20.2 & 40.0 & 77.0 & 151.9 \\
\hline 4 & $(3,6.0)$ & 7.9 & 15.0 & 28.9 & 56.9 & 113.7 \\
\hline 5 & $(3,8.5)$ & 7.1 & 13.0 & 24.7 & 48.4 & 96.6 \\
\hline
\end{tabular}

increased. Then, there exists an optimal $(p, s)$ pair satisfying the desired level of accuracy with the minimum processing time. We scan the parameters $p$ and $s$ for various values of $a$ and $d_{0}$. All possible values of $D$ according to the one-box-buffer scheme [6] are also checked. In the end, we obtain the optimal values listed in Table I with the corresponding speedup compared to the direct calculation. We note that the values presented in Table I do not depend on the computer platform. The optimal $(p, s)$ pairs are valid for $a \geq 4 \lambda$ and they are found to be independent of $D$. For smaller clusters, such as $a=\lambda$ or $2 \lambda$, the interpolation does not lead to a significant speedup, and therefore, we prefer to calculate these translations directly. In the case of much smaller clusters, such as $a=0.25 \lambda$ or $0.5 \lambda$, direct calculation is faster than the interpolation for any $(p, s)$ pair satisfying the desired accuracy.

Fig. 4(a) and (b) compares the optimal $(p, s)$ pairs to the fixed $p=3, s=5.0$ values suggested in [4]. In Fig. 4(a), the interpolation error is plotted with respect to the box size $a$ from $4 \lambda$ to $64 \lambda$ and for different levels of accuracy, i.e., for $d_{0}=2,3,4$, and 5 corresponding to $10^{-2}, 10^{-3}, 10^{-4}$, and $10^{-5}$ relative errors, respectively. In the optimized case, the error is always below the desired level of accuracy. However, with fixed parameters, the error is not controllable and is localized around $10^{-4}$. The corresponding speedup is plotted in Fig. 4(b), where it increases with increasing box size and decreases with increasing number of accurate digits in the optimized case. This relationship is also evident in Table I. Comparing Fig. 4(a) and Fig. 4(b), the following observations can be made.

1) For $d_{0}=2$ and 3, fixed $p=3, s=5.0$ satisfies the desired level of accuracy but the optimal $(p, s)$ pairs provide higher speedup.

2) For $d_{0}=4$ and 5, the fixed $p=3, s=5.0$ seems to give higher speedup compared to the optimal $(p, s)$ pairs, however, the accuracy is not satisfied with the fixed parameters.

Based on these observations, we conclude that optimization is essential to improve the interpolation of the translation operator.

\section{RESULTS}

To demonstrate the overall improvement obtained with interpolation, we present the results of a scattering problem involving a conducting sphere of radius $20 \lambda$. This is a $1,462,854-$ unknown problem solved by a parallel MLFMA implementation with seven levels. The problem is solved on a cluster of 32 2.6-GHz Pentium-4 Celeron processors. The box size is $0.25 \lambda$ for the lowest level and $16 \lambda$ for the highest level. As an example, if the number of accurate digits $d_{0}$ is set to 3 , then $L$ takes values from 8 to 195 . We use the one-box-buffer scheme and reduce the number of translations by exploiting the symmetry [7]. During the setup phase of the program, each processor checks all of its 

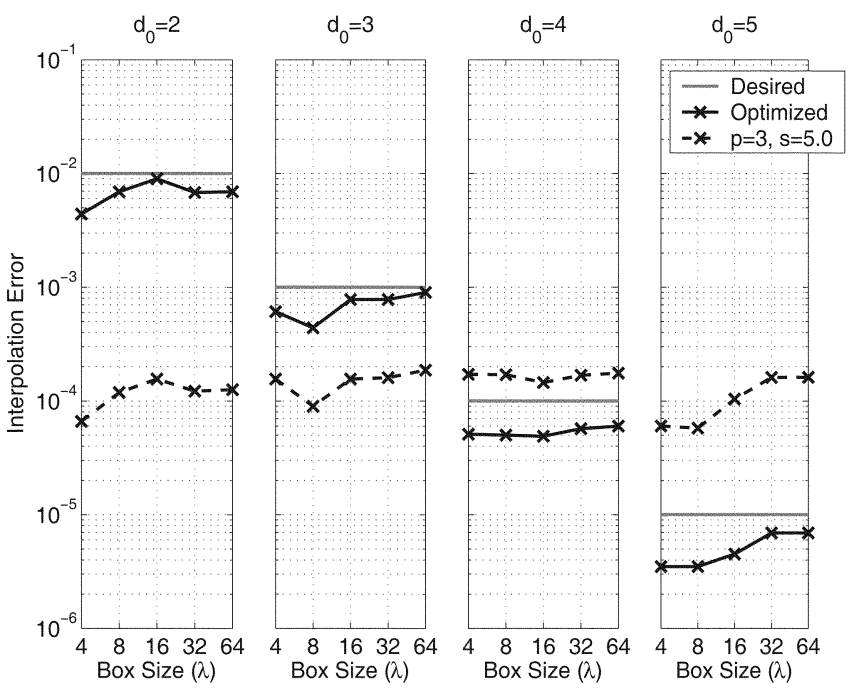

(a)
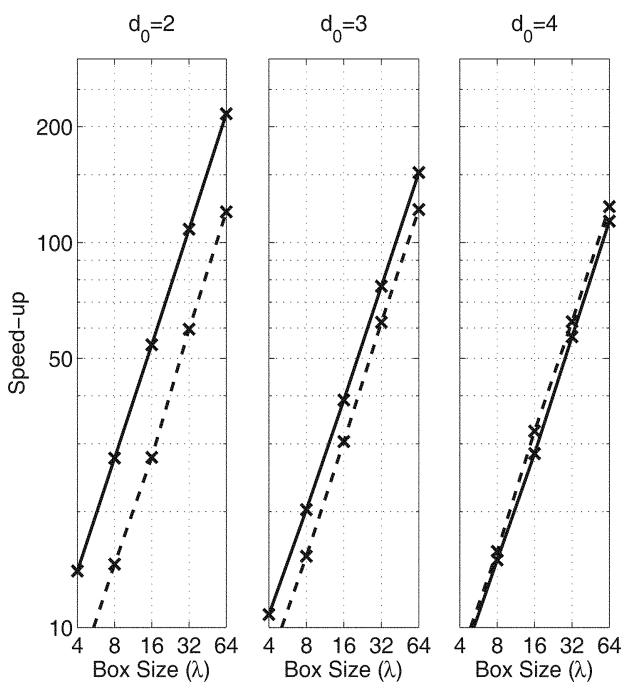

(b)

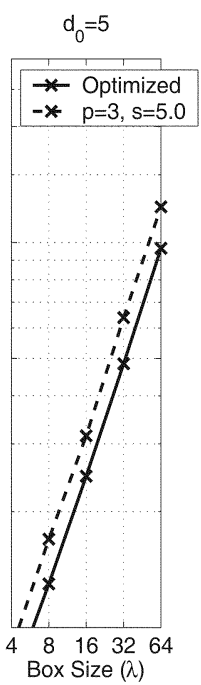

Fig. 4. (a) Interpolation error and (b) corresponding speedup for different box sizes from $4 \lambda$ to $64 \lambda$ and for $d_{0}=2,3,4,5 .(\boldsymbol{D}=\hat{\boldsymbol{x}} 2 a)$.

cluster-cluster interactions to eliminate the unneeded translations.

In Fig. 5(a), processing time for the calculation of the translation operators is plotted with respect to $d_{0}$. For both types of calculations (direct and interpolated), the maximum is chosen among the processing times spent by 32 processors. In Fig. 5(b), the speedup obtained by the interpolation method over direct calculation is plotted as a function of $d_{0}$. The speedup is over 14 up to $d_{0}=5$.

\section{CONCLUSION}

In this paper, we revisited the Lagrange interpolation of the translation operator in 3-D MLFMA. We optimized the number of interpolation points $p$ and the oversampling factor $s$. In this way, the error becomes controllable and the processing time required to satisfy the desired level of accuracy is minimized.

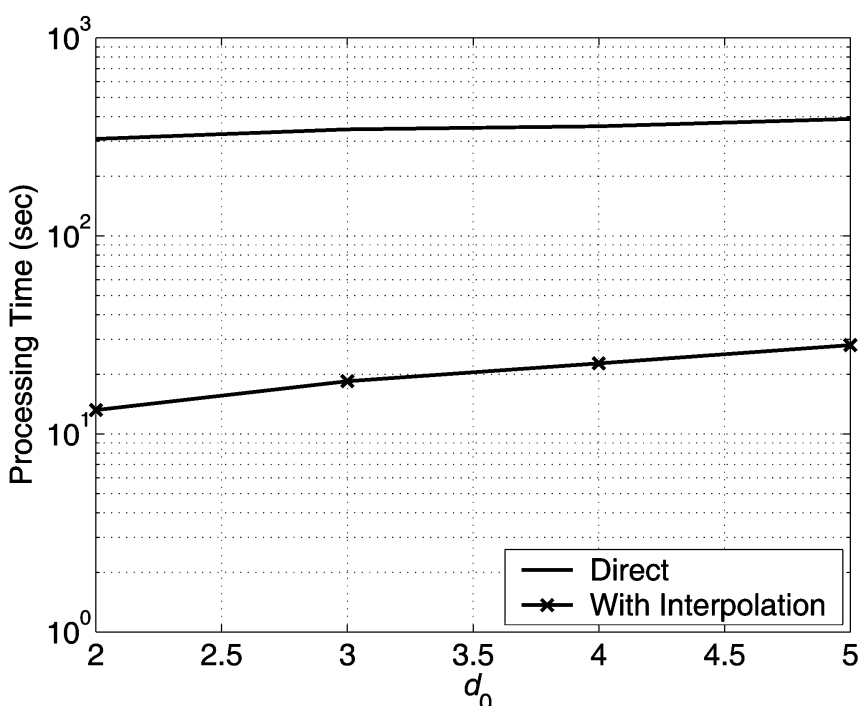

(a)

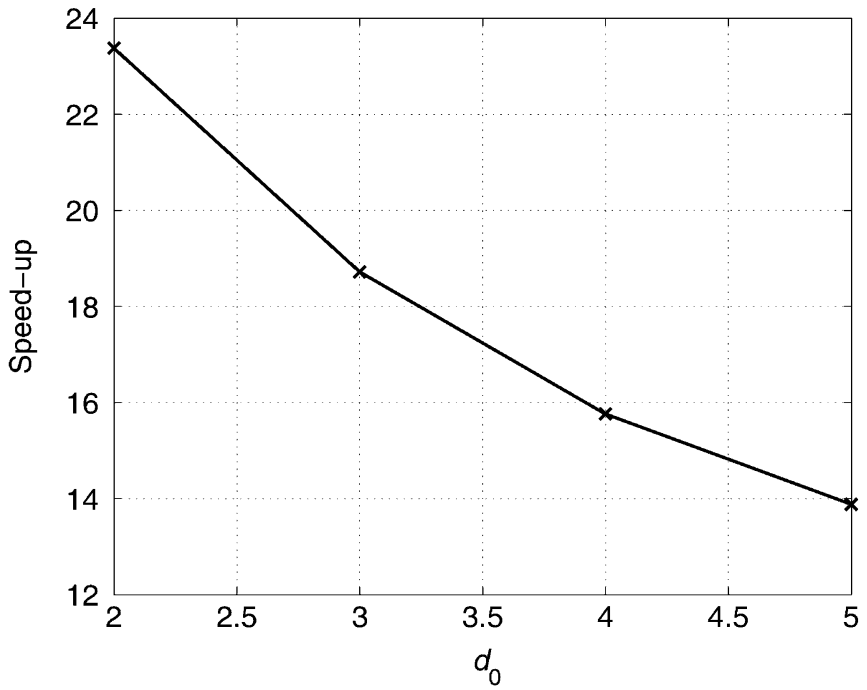

(b)

Fig. 5. (a) Processing time to compute the translation operators for a $1,462,854$ unknown sphere problem. (b) Speedup obtained with optimal interpolation compared to direct calculation of the translation operators.

\section{REFERENCES}

[1] W. C. Chew, J.-M. Jin, E. Michielssen, and J. Song, Fast and Efficient Algorithms in Computational Electromagnetics. Boston, MA: Artech House, 2001.

[2] C.-C. Lu and W. C. Chew, "Multilevel fast multipole algorithm for electromagnetic scattering by large complex objects," IEEE Trans. Antennas Propag., vol. 45, no. 10, pp. 1488-1493, Oct. 1997.

[3] R. Coifman, V. Rokhlin, and S. Wandzura, "The fast multipole method for the wave equation: A pedestrian prescription," IEEE Antennas Propag. Mag., vol. 35, no. 3, pp. 7-12, Jun. 1993.

[4] J. Song and W. C. Chew, "Interpolation of translation matrix in MLFMA," Microwave Opt. Technol. Lett., vol. 30, no. 2, pp. 109-114, Jul. 2001.

[5] S. Koc, J. M. Song, and W. C. Chew, "Error analysis for the numerical evaluation of the diagonal forms of the scalar spherical addition theorem," SIAM J. Numer. Anal., vol. 36, no. 3, pp. 906-921, 1999.

[6] M. L. Hastriter, S. Ohnuki, and W. C. Chew, "Error control of the translation operator in 3D MLFMA," Microwave Opt. Technol. Lett., vol. 37, no. 3, pp. 184-188, May 2003

[7] S. Velamparambil, W. C. Chew, and J. Song, "10 million unknowns: Is that big?," IEEE Antennas Propag. Mag., vol. 45, no. 2, pp. 43-58, Apr. 2003. 


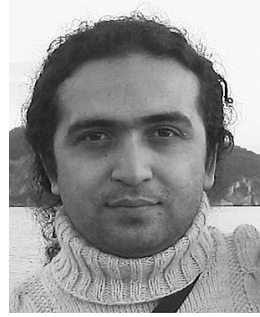

Özgür Ergül (S'98) was born in Yozgat, Turkey, in 1978. He received the B.S. and M.S. degrees in electrical and electronics engineering from Bilkent University, Ankara, Turkey, in 2001 and 2003, respectively. He is currently pursuing the Ph.D. degree at Bilkent University.

Since 2001, he has served as a Teaching and Research Assistant in the Department of Electrical and Electronics Engineering at Bilkent University. From 2000 to 2005 , he was affiliated with the Computational Electromagnetics Research Center (BilCEM). His research interests include fast and accurate algorithms for the solution of large and complicated structures, parallel programming, and iterative techniques.

Mr. Ergül's academic endeavors are supported by the Scientific and Technical Research Council of Turkey (TUBITAK) in the framework of a national Ph.D. scholarship.

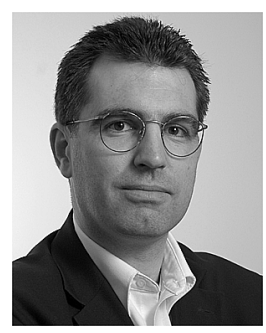

Levent Gürel (S'87-M'92-SM'97) received the B.Sc. degree from the Middle East Technical University (METU), Ankara, Turkey, in 1986 and the M.S. and Ph.D. degrees from the University of Illinois at Urbana-Champaign (UIUC), Urbana, in 1988 and 1991, respectively, all in electrical engineering.

He joined the IBM Thomas J. Watson Research Center, Yorktown Heights, NY, in 1991, where he worked as a Research Staff Member on the electromagnetic compatibility (EMC) problems related to electronic packaging, on the use of microwave processes in the manufacturing and testing of electronic circuits, and on the development of fast solvers for interconnect modeling. Since 1994, he has been a faculty member in the Department of Electrical and Electronics Engineering of the Bilkent University, Ankara, where he is currently a Professor. He was a Visiting Associate Professor at the Center for Computational Electromagnetics (CCEM) of the UIUC for one semester in 1997. He returned to the UIUC as a Visiting Professor during 2003-2005, and as an Adjunct Professor during 2005-2006. He founded the Computational Electromagnetics Research Center (BiLCEM) at Bilkent University in 2005, where he is serving as the Director. His research interests include the development of fast algorithms for computational electromagnetics (CEM) and the application thereof to scattering and radiation problems involving large and complicated scatterers, antennas, and radars; frequency-selective surfaces; high-speed electronic circuits; optical and imaging systems; nanostructures; and metamaterials. He is also interested in the theoretical and computational aspects of electromagnetic compatibility and interference analyses. Ground penetrating radars and other subsurface scattering applications are also among his research interests.

Among the recognitions of Prof. Gürel's accomplishments, the two prestigious awards from the Turkish Academy of Sciences (TUBA) in 2002 and the Scientific and Technical Research Council of Turkey (TUBITAK) in 2003 are the most notable. He served as the Chairman of the AP/MTT/ED/EMC Chapter of the IEEE Turkey Section from 2000 to 2003. He founded the EMC Chapter in Turkey in 2000. He served as the Co-Chairman of the 2003 IEEE International Symposium on Electromagnetic Compatibility. He is a member of the General Assembly of the European Microwave Association, a member of the USNC of the International Union of Radio Science (URSI), and the Chairman of Commission E (Electromagnetic Noise and Interference) of URSI Turkey National Committee. He is currently serving as an Associate Editor of Radio Science. 